\title{
Translocation of gliadin into HLA-DR antigen containing lysosomes in coeliac disease enterocytes
}

\author{
K-P Zimmer, C Poremba, P Weber, P J Ciclitira, E Harms
}

\begin{abstract}
Coeliac disease is triggered by ingestion of wheat gliadin and is probably immune mediated. There is evidence by light microscopy that expression of class II major histocompatibility complex (MHC) molecules is increased in the small intestinal epithelium of patients with untreated coeliac disease and that gliadin can be taken up by small intestinal enterocytes. The pathway by which gliadin is transported to class II MHC proteins has not been demonstrated. Using an immunogold technique and thin frozen sections of jejunal biopsy specimens, gliadin, HLA-DR antigens, and IgA were localised at an ultrastructural level in the jejunal epithelium of patients with both untreated and treated coeliac disease and controls. Cathepsin D was used as a marker for late endosomes or lysosomes. The results show that gliadin is translocated into vacuoles positive for HLA-DR antigens as well as cathepsin $D$ in jejunal enterocytes of patients with untreated coeliac disease. Secretory IgA may have a role in this translocation of gliadin, which is a specific event that occurred only in jejunal enterocytes from patients with untreated coeliac disease but not in a patient maintained on a gluten free diet or in controls. These results support a central role for epithelial cells of the human intestinal mucosa in the transport of gliadin to an HLA-DR positive compartment which precedes antigen presentation of gliadin to antigen sensitive T lymphocytes.

(Gut 1995; 36: 703-709)
\end{abstract}

Keywords: endocytosis of gliadin, cathepsin D, immunocytochemistry, secretory IgA, antigen presentation.

University Children's

Hospital, 48149

Münster, Germany

K-P Zimmer

C Poremba

P Weber

E Harms

Gastroenterology Unit, UMDS, St Thomas's Hospital, London SE1 7EH

P J Ciclitira

Correspondence to: Dr P Zimmer, Klinik und Poliklinik für

Kinderheilkunde, Universität Münster, Albert-SchweitzerStrasse 33, 48149 Münster, Germany.

Accepted for publication 30 August 1994 malabsorption of most nutrients, is thought to be immune mediated. ${ }^{12}$ Gliadin, the alcohol soluble component of wheat gluten exacerbates the condition. A gluten free diet leads to the regression of both the clinical symptoms and abnormalities present in the mucosa of the small intestine. The pathogenetic mechanisms of the disorder remain unknown, although genetic and immune factors have been identified. $^{3}$ Experiments involving light microscopy recently showed that in vivo uptake of a peptic tryptic digest of gliadin is greater in human enterocytes of untreated coeliac disease patients than in control subjects. ${ }^{4}$

It has been shown with cell culture systems that the immune response to microbial antigens as well as autoantigens was initiated by the presentation of exogenous peptides to $\mathrm{T}$ lymphocytes. This involves binding of endocytosed and processed peptides to class II major histocompatibility complex (MHC) molecules. ${ }^{5}$ It has been suggested that the association of processed antigens and class II MHC proteins takes place in the endosomal ${ }^{7}$ or lysosomal ${ }^{89}$ compartments.

Exogenous antigen presentation is mediated by activated $B$ cells, myeloid cells, and dendritic cells and remains to be demonstrated in epithelial cells. ${ }^{5}$ The increased expression of class II MHC proteins ${ }^{10-12}$ as well as the detection by light microscopy of endocytosed gliadin in the intestinal epithelium of patients with coeliac disease 4 suggest that antigen presentation of gliadin occurs in enterocytes. The precise subcellular localisation of endocytosed gliadin remains to be determined. We wished to determine the cell type and the subcellular structures associated with colocalisation of gliadin and class II MHC proteins in the small intestine of individuals with coeliac disease. We also wished to determine whether factors such as secretory IgA and HLA-DR antigens are involved in the uptake of gliadin. Finally, we wished to find out if the subcellular co-localisation of gliadin and HLA-DR antigens is specific to patients with untreated coeliac disease.

We used an immunogold technique on thin frozen sections of small bowel biopsy tissue to demonstrate that gliadin is localised in HLA-DR antigen positive lysosomes of small intestinal epithelial cells of patients with untreated coeliac disease. Our experiments indicate that this co-localisation is due to a specific transport of gliadin which may be a prerequisite for the antigen presentation of gliadin and the immune reactions observed in this condition.

\section{Methods}

COELIAC DISEASE PATIENTS

Jejunal biopsy specimens were obtained from six patients who presented with untreated coeliac disease. The diagnosis in these patients was subsequently confirmed by ESPGAN criteria. ${ }^{13}$ The biopsy specimens exhibited villous atrophy on histological examination. 
After treatment with a strict gluten free diet, all six subjects showed symptoms of remission and improvement in the villous morphology of a subsequent jejunal biopsy specimen. One biopsy specimen from a patient with coeliac disease who had received a gluten free diet for three years was studied also. Jejunal biopsy specimens from three subjects with normal villous morphology, all of whom were subsequently diagnosed as suffering from the irritable bowel syndrome served as controls.

\section{ANTIBODIES}

We used rabbit antibodies against gliadin (Sigma, dilution $1: 100) ; \alpha$ gliadin, ${ }^{14}$ $\beta$ gliadin, ${ }^{14} \gamma$ gliadin, ${ }^{14} \omega$ gliadin ${ }^{14}$ (dilutions 1:500); cathepsin D (Dako, dilution 1:40); bovine albumin (Sigma, dilution 1:50); chicken egg albumin (Sigma, dilution 1:50); biotinylated rabbit $F\left(a b^{\prime}\right)_{2}$ fragments against the $\alpha$ chain of human IgA (Dianova, dilution $1: 30)$; and mouse monoclonal antibodies against HLA-DR (Dako, dilution 1:40) and HLA-DR (Dianova, dilution 1:40), the last two being pooled. Binding sites were visualised by gold conjugated goat anti-rabbit anti-serum (diameter of $6 \mathrm{~nm}$ and $12 \mathrm{~nm}$, Dianova, dilution 1:50); gold conjugated goat antimouse anti-serum (diameter of $6 \mathrm{~nm}$, Dianova, dilution 1:10); and streptavidin gold (diameter of $10 \mathrm{~nm}$, Amersham, dilution 1:40). The streptavidin-biotin reaction was used to avoid cross labelling of the second immunogold with the antibodies of the first labelling step. A goat antibody against human $\operatorname{IgA}, \operatorname{IgG}$, and $\operatorname{IgM}$ (Dianova, dilution 1:10) served to block the binding of biotinylated rabbit antibody against human IgA and to show that the labelling of gliadin, HLA-DR proteins, and cathepsin D was not caused by cross reactivity of these antibodies against human immunoglobulins present in the intestinal lumen and epithelium.

EMBEDDING OF BUFFY COAT MACROPHAGES IN GLIADIN/GELATINE

Buffy coat macrophages were fixed in $5 \%$ paraformaldehyde. Enzymatic digestion of gliadin (Sigma) by pepsin (Sigma) and trypsin (Sigma) was carried out as described elsewhere. ${ }^{4}$ Buffy coat macrophages were embedded in the peptic tryptic digest of gliadin mixed $1: 1$ with $10 \%$ gelatine in phosphate buffered saline.

\section{IMMUNOELECTRON MICROSCOPY}

Small bowel biopsy and buffy coat macrophages embedded on gelatine/gliadin were prepared for sectioning and labelling of thin-frozen sections using the technique of Tokuyasu and Griffiths ${ }^{15} 16$ as described previously. ${ }^{17}$ Briefly, small tissue specimens were fixed in $5 \%$ paraformaldehyde in $50 \mathrm{mM}$ Hepes, $\mathrm{pH} \quad 7 \cdot 4$, cryoprotected by polyvinylpyrrolidone/sucrose, frozen in liquid nitrogen, and sectioned with a Sorvall cryoultramicrotome MT 6000 at $-100^{\circ} \mathrm{C}$. Cryosections were incubated at room temperature with antibodies for 45 minutes. Having been labelled with immunogold, the grids were contrasted, embedded in $2 \%$ methylcellulose (1 ml methylcellulose contained $0.1 \mathrm{ml} \mathrm{3 \%}$ uranyl acetate), and examined in a Philips 301 electron microscope.

\section{Results}

\section{ENDOCYTOSIS OF GLIADIN BY ENTEROCYTES}

Labelling of thin frozen sections of jejunal mucosal biopsy specimens from patients with untreated coeliac disease with antibodies against gliadin, $\alpha$ gliadin, $\beta$ gliadin, $\gamma$ gliadin, and $\omega$ gliadin showed the following patterns. A low amount of gliadin is present in the lumen of tubular and vesicular structures close to the apical membrane of enterocytes characterised by microvilli (not shown). In addition, high concentrations of gliadin are present in large endocytic vacuoles contained within the enterocytes (Fig 1A) and the intestinal lumen. The labelling density of gliadin was much stronger inside the large endocytic vacuoles than on the luminal site of the apical

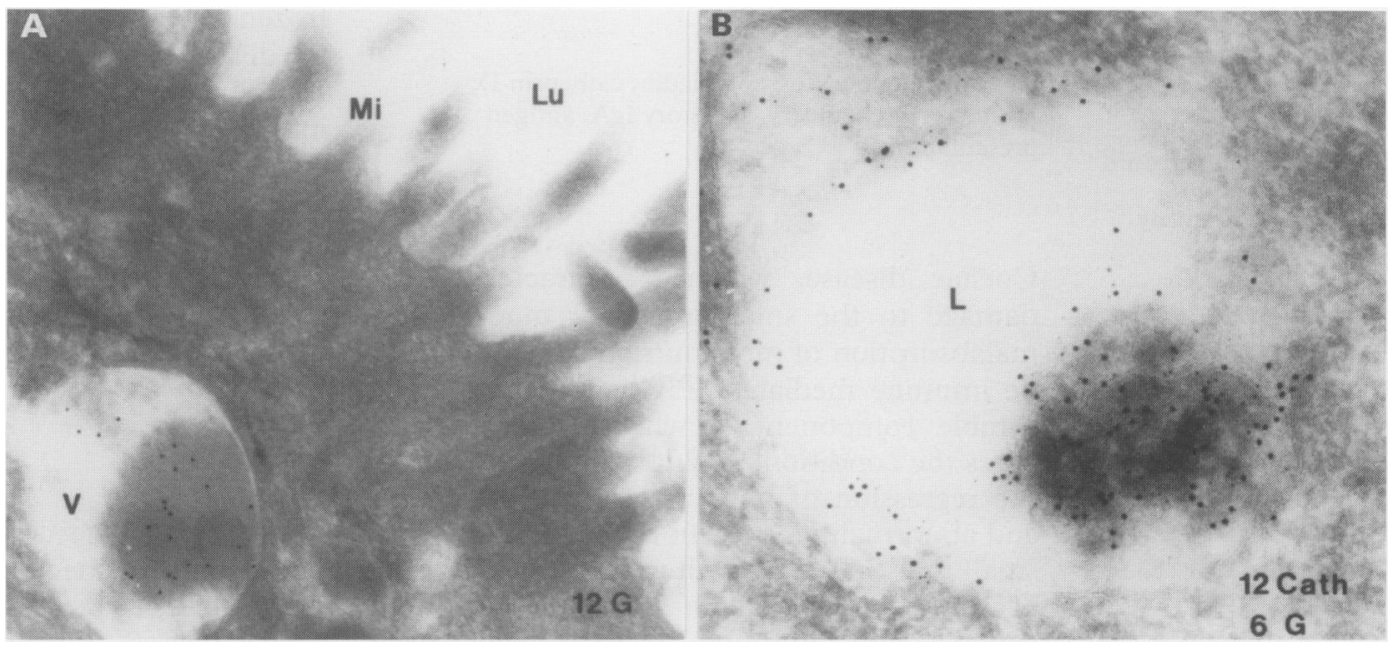

Figure 1: Uptake of gliadin into lysosomes in gut epithelial cells of untreated coeliac disease patients. (A) Gliadin $(G 12 \mathrm{~nm})$ is present in a vacuole $(V)$ close to the apical membrane of enterocytes. Lu=luminal site of the apical membrane; Mi=microvilli. (Magnification $\times 56000$.) (B) Gliadin $(G 6 \mathrm{~nm}$ ) accumulates in compartment containing cathepsin D (Cath $12 \mathrm{~nm})$. L=lysosome. (Magnification $\times 74$ 000.) 

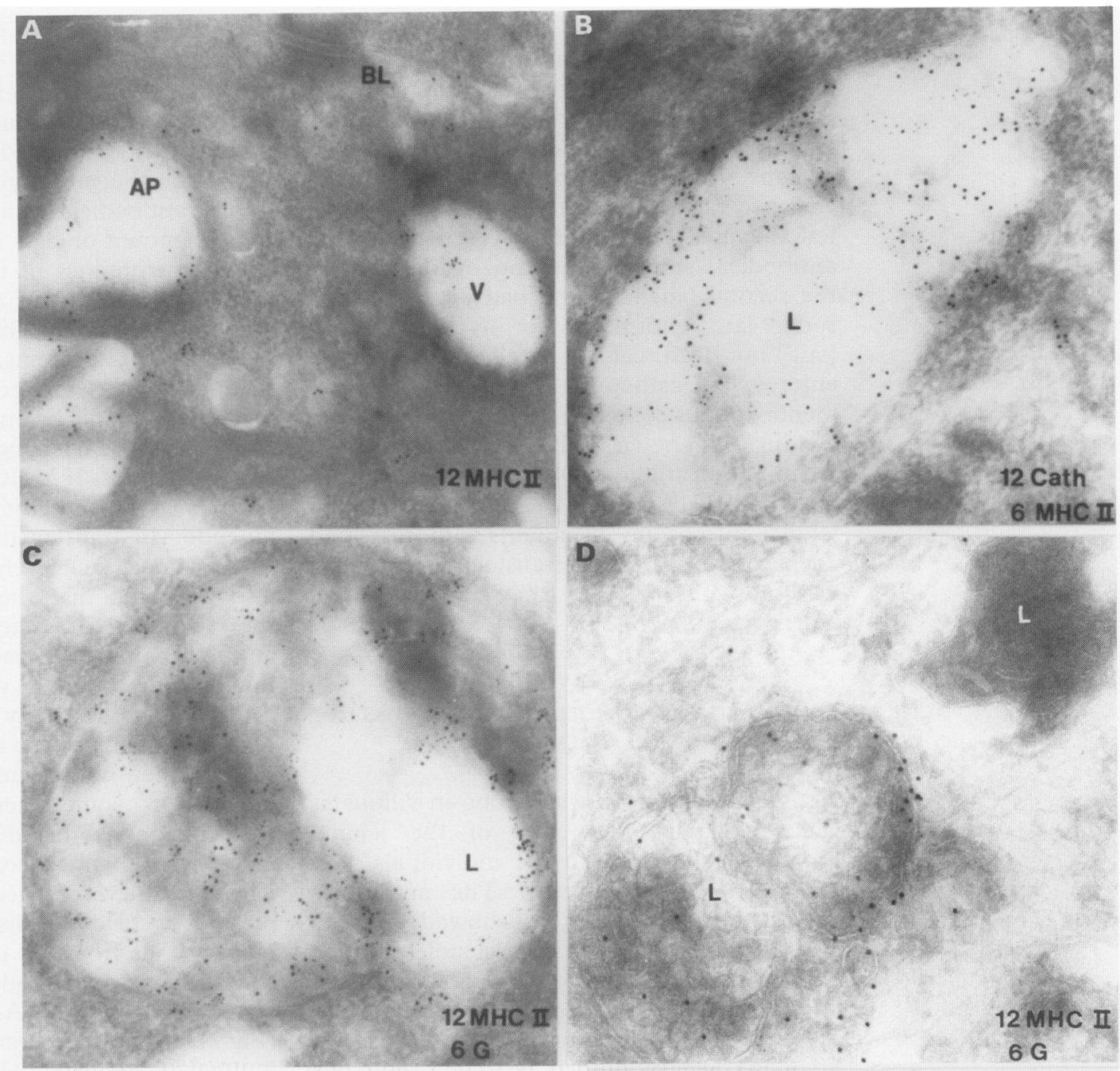

E

Figure 2: Accumulation of gliadin in vacuoles containing HLA-DR antigens in enterocytes of patients with active coeliac disease. (A) Strong staining of vacuoles $(V)$ and the apical membrane (AP) with HLA-DR antigens (MHC II $12 \mathrm{~nm}$ ) in coeliac disease enterocytes. $B L=$ basolateral membrane. (Magnification $\times 32$ 000.) (B) Localisation of HLA-DR antigens (MHC II $6 \mathrm{~nm}$ ) in cathepsin D (Cath $12 \mathrm{~nm}$ ) positive compartments of enterocytes. L=lysosome. (Magnification X64 000.) C) Co-localisation of gliadin $(G 6 \mathrm{~nm})$ and $H L A-D R$ antigens (MHC II $12 \mathrm{~nm}$ ) in enterocytes of untreated coeliac disease patients. (Magnification $\times 57$ 000.) (D) Enterocyte of a patient with coeliac disease in remission shows vacuoles with HLA-DR antigens (MHC II $12 \mathrm{~nm}$ ) but without any presence of gliadin $(G 6 \mathrm{~nm})$. L=lysosome. (Magnification $\times 74$ 000.) (E) Buffy coat macrophages, embedded in the mixture of gliadin and gelatine, indicated no immunogold binding of the second labelling step (antibodies against HLA-DR antigens: MHC II $12 \mathrm{~nm}$ ) to the antibodies of the first labelling step (antibody against gliadin: $G 6 \mathrm{~nm}) . N=$ nucleus; $P M=$ plasma membrane; $E X=$ extracellular.

membrane. The labelling patterns of the antibodies directed against the four gliadin fractions were similar. $M$ cells, which, it has been suggested, are involved in antigen uptake and presentation to local T lymphocytes, could not be found in any of our jejunal biopsy specimens. In contrast to the small intestinal biopsy specimens from the patient with untreated coeliac disease, those from the controls showed few gliadin molecules, present only in the tubular and vesicular structures of those enterocytes close to the apical membrane. Large endocytic vacuoles throughout enterocytes with a high amount of gliadin could not be identified in these specimens (not shown).

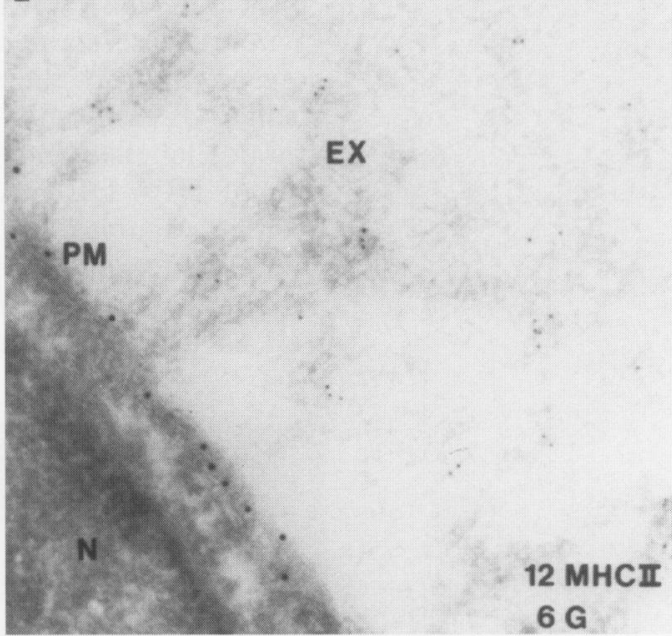

The jejunal biopsy specimen from the single patient with treated coeliac disease who had taken a gluten free diet did not reveal gliadin in any of those enterocytes examined. These results suggest that gliadin can be endocytosed by jejunal enterocytes of individuals with coeliac disease as well as controls but that the localisation and amount in the endocytic apparatus differs in these patient groups.

CATHEPSIN D IN GLIADIN ENRICHED VACUOLES OF COELIAC DISEASE ENTEROCYTES

Cathepsin D, which is a marker of lysosomes and late endosomes, was used to characterise 
further the enterocyte compartment of the jejunal enterocytes from the untreated coeliac patients where gliadin was concentrated. Sections labelled with the antibody against gliadin as well as cathepsin D showed that cathepsin D is present inside the gliadin enriched vacuoles (Fig 1B). We could identify few vacuoles stained only by one of the added antibodies, showing that no overlapping of the second labelling step had occurred, and some late endosomes or lysosomes had (still) not been reached by gliadin. In control enterocytes, cathepsin D positive compartments did not contain a noticeable amount of gliadin (not shown). The double labelling of gliadin and cathepsin $\mathrm{D}$ indicates that gliadin is routed to lysosomes or late endosomes in jejunal enterocytes of patients with untreated coeliac disease. This translocation process was not observed in the control jejunal enterocytes.

CO-LOCALISATION OF GLIADIN WITH CLASS II MHC PROTEINS IN COELIAC DISEASE

ENTEROCYTES

HLA-DR expression was strong in villous as well as crypt enterocytes of the jejunal biopsy specimens from the individuals with untreated coeliac disease. The antibodies against HLA-DR antigens showed appreciable staining in endocytic vacuoles, which was stronger than that observed at the apical and basolateral membrane of the enterocytes (Fig 2A). In control biopsy tissues, HLA-DR expression was restricted to villous epithelial cells, where fewer endocytic vacuoles were stained in comparison to the enterocytes from the subjects with untreated coeliac disease and the apical membrane was not labelled (not shown). A co-localisation of HLA-DR molecules in cathepsin D positive vacuoles could be observed in the jejunal mucosal biopsy specimens from the patients with untreated coeliac disease as well as in those from controls, although it was more frequent in the former group (Fig 2B).

Double labelling experiments with thin frozen sections of jejunal biopsies from the patients with untreated coeliac disease, using an antibody against gliadin followed by the pooled antibodies against HLA-DR, exhibited a high amount of class II MHC proteins close to the membranes of the endocytic vacuoles enriched with gliadin (Fig 2C). Besides the structures which exhibited both gliadin and class II MHC proteins, we were also able to identify vesicles on the same sections which were labelled only by one of the two antibodies (not shown). This suggested that gliadin did not reach a class II MHC protein positive compartment inside enterocytes before performing an endocytic translocation process. The structures stained exclusively by the first antibody also indicated that the antibodies added in the second labelling step did not bind to the antibodies of the first labelling step, excluding non-specific binding of the labelling procedure. There was only a weak co-localisation of both proteins on the apical membrane.

Only a few compartments with little gliadin as well as some vacuoles with HLA-DR molecules could be identified in the jejunal enterocytes obtained from the control subjects. These cells never showed a vacuole where both proteins were co-localised (not shown). The biopsy tissue from the individual with treated coeliac disease who had been on a gluten free diet showed only HLA-DR positive vacuoles (Fig 2D). These experiments show that a high number of gliadin and HLA-DR molecules can accumulate in jejunal enterocyte lysosomes in subjects with untreated coeliac disease, but not in controls.

PRESENCE OF IgA IN VACUOLES ENRICHED WITH CLASS II MHC PROTEINS OR GLIADIN

A high amount of IgA was localised with gliadin in the luminal side of the apical membrane in the jejunal biopsy specimens

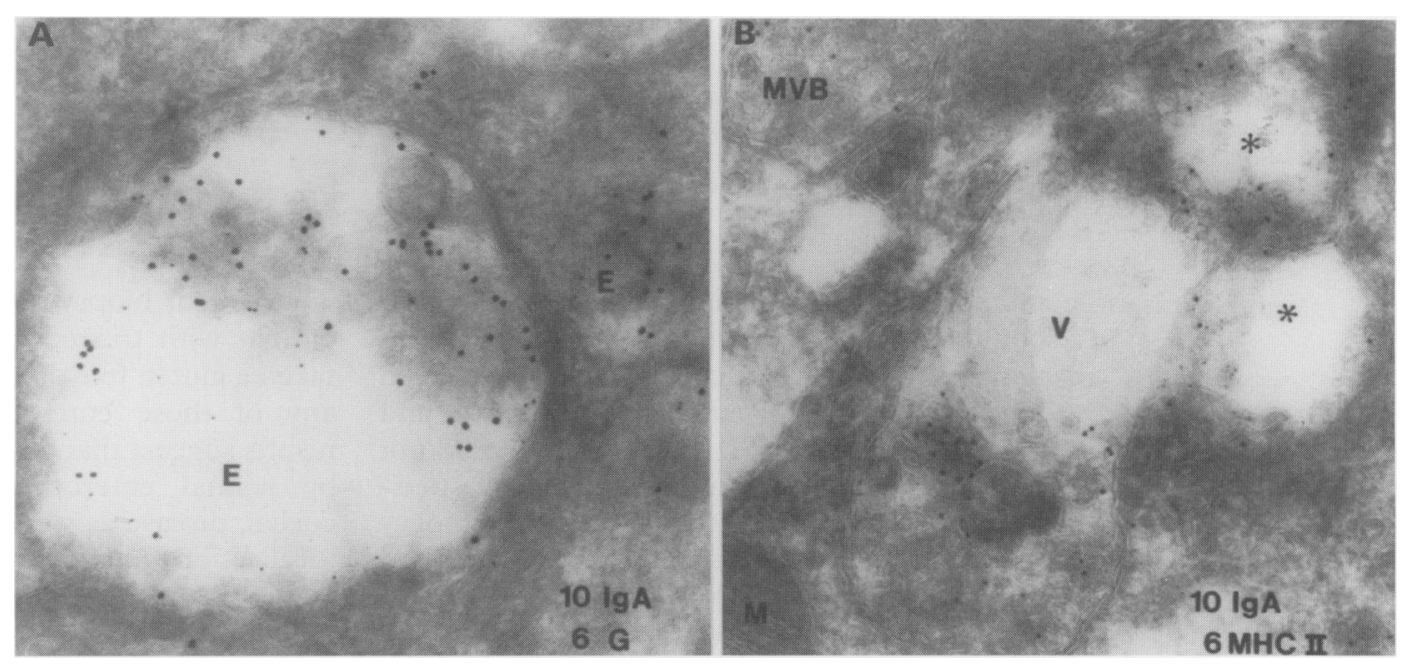

Figure 3: Presence of IgA in endocytic vacuoles observed in gut epithelial cells of patients with active coeliac disease.

(A) Double labelling of gliadin $(G 6 \mathrm{~nm})$ and $\mathrm{Ig} A(10 \mathrm{~nm})$ shows $\mathrm{Ig} A$ inside a gliadin containing compartment. The high IgA and low gliadin suggest that this endocytic vacuole is an endosome. $E=$ endosome. (Magnification $\times 65000$.) (B) The compartment enriched with HLA-DR antigens (MHC II $6 \mathrm{~nm})$ also contains IgA $(10 \mathrm{~nm})$. The high concentration of class II MHC proteins points to the lysosomal nature of the vacuole. $M V=$ multivesicular body; $M=$ mitochondrium; $V=v a c u o l e$. *Indicates parts of the vacuole with co-localisation of IgA and class II MHC proteins. (Magnification $\times 54000$.) 


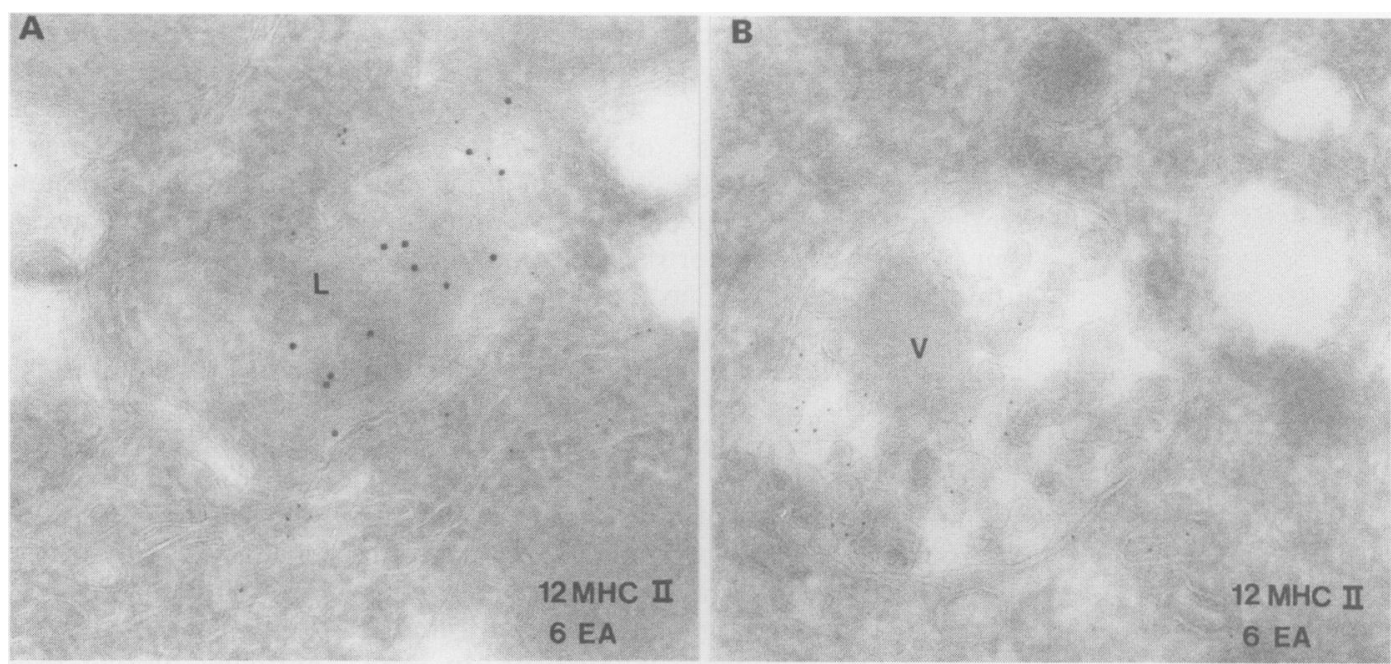

Figure 4: Localisation of chicken egg albumin and HLA-DR antigens in enterocytes of patients with active coeliac disease. Chicken egg albumin and $H L A-D R$ antigens are present in different cellular compartments. ( $A$ ) Endocytic vacuole containing HLA-DR antigens (MHC II $12 \mathrm{~nm}$ ) and lacking chicken egg albumin (EA $6 \mathrm{~nm}$ ), which is only detectable outside the vacuole. The feature is in contrast to the strong co-localisation of gliadin and HLA-DR antigens shown in Figure 2(C). A few chicken egg albumin molecules associated with HLA-DR antigens may be caused by background staining. Alternatively, they may indicate that the spatial separation of both proteins is not absolute. $L=l y$ sosome. (Magnification $\times 93000$.) (B) This endocytic vacuole $(V)$, filled with chicken egg albumin (EA $6 \mathrm{~nm})$, is situated close to the organelle shown in $(A)$ in the same cell. Although this ultra-thin frozen section is labelled with antibodies against $H L A-D R$ antigens (which are indicated in $(A))$, there are no HLA-DR antigens (MHC II $12 \mathrm{~nm}$ ) present in this organelle. (Magnification $\times 90000$.)

from the patients with untreated coeliac disease as well as in control enterocytes. In jejunal biopsy tissues of coeliac patients eating a gluten containing diet, IgA could be detected in endocytic vacuoles which contained a minor amount of gliadin (Fig 3A). In the same enterocytes, we also found gliadin enriched vacuoles with a weaker but appreciable labelling of IgA (not shown). Simultaneous labelling experiments also detected noticeable amounts of IgA in class II MHC protein enriched compartments of jejunal enterocytes from the untreated coeliac patients (Fig 3B). The co-localisation of IgA in compartments enriched with class II MHC protein or gliadin was not observed in any of the control enterocytes (not shown).

\section{CONTROL EXPERIMENTS}

No background labelling was seen either in nuclei or mitochondria with the antibody dilutions used. Sections labelled with gold conjugated goat anti-rabbit anti-serum and goat anti-mouse anti-serum as well as streptavidin gold alone showed no appreciable staining. Chicken egg and bovine albumin were found in vacuoles of jejunal enterocytes from subjects with untreated coeliac disease and controls, but no co-localisation with HLADR antigens was observed. Figure 4A shows an endocytic vacuole containing HLA-DR antigens that lack chicken egg albumin, which is only outside the vacuole. This feature contrasts with the strong co-localisation of gliadin and HLA-DR antigens shown in Figure 2C. Figure 4B shows an endocytic vacuole $(V)$, filled with chicken egg albumin which is situated close to the organelle shown in Figure 4A. Although this ultra-thin frozen section was labelled with antibodies against HLA-DR antigens which are indicated in Figure 4A, there were no HLA-DR antigens present in this organelle.

Preincubation with the goat antibody against human IgA, IgG, and IgM blocked the binding of biotinylated rabbit antibody against human IgA, but the labelling of gliadin, HLA-DR proteins, and cathepsin D was not affected by this procedure. When the double labelling procedure for the co-localisation of gliadin and HLA-DR antigens was performed on buffy coat macrophages, embedded in the mixture of gliadin and gelatine, gliadin was seen in the extracellular space and class II MHC proteins exclusively on the plasma membrane of the macrophages (Fig 2E). The last experiment indicated that the colocalisation of gliadin and HLA-DR antigens in the enterocytes from subjects with untreated coeliac disease was not caused by cross reaction of the antibodies of the second labelling step with those in the first labelling step.

\section{Discussion}

Immunohistochemical studies of small intestinal epithelium from patients with untreated coeliac disease suggest that gliadin is taken up by enterocytes. ${ }^{4}$ Our results confirm these results obtained by light microscopy (Fig 1A). In addition, we note an accumulation of gliadin in cathepsin $\mathrm{D}$ containing vacuoles of these enterocytes indicating that gliadin can be translocated to late endosomes or lysosomes from patients with untreated coeliac disease (Fig 1B). In contrast to the enterocytes from patients with untreated coeliac disease, we could not detect gliadin in late endosomes or lysosomes of control enterocytes. In addition, gliadin was not observed in jejunal enterocytes from a patient with treated coeliac disease who had been taking a gluten free diet (Fig 2D). 
Other immunohistochemical experiments show that the class II MHC protein expression is increased in villous as well as crypt small intestinal enterocytes of patients with untreated coeliac disease. ${ }^{1011}$ This enhanced expression can be induced by the administration of gliadin. ${ }^{12}$ In normal intestinal epithelium, the class II MHC expression is restricted to the villous epithelium. ${ }^{1011}$ Using the immunoperoxidase technique and electron microscopy, class II MHC proteins are reported to be present in multivesicular bodies, secondary lysosomes, and the basolateral as well as the apical membrane of normal human enterocytes. ${ }^{18}$ Our experiments show a strong co-localisation of HLA-DR antigens with cathepsin $\mathrm{D}$ indicating that the main amount of HLA-DR antigens is present in late endosomes or lysosomes (Fig 2B). The apical membrane of enterocytes from patients with untreated coeliac disease contains HLA-DR antigens which are not observed in control subjects (Fig 2A). Class II MHC proteins are believed to be mainly transported from the Golgi apparatus directly to a lysosomal compartment in lymphocytes. ${ }^{8}$ The presence of HLA-DR antigens at the apical membrane may be due to their enhanced expression. This phenomenon is known from other proteins such as lysosomal membrane proteins. A part of these proteins, which are known to be transported directly to late endosomes and lysosomes without appearing on the cell surface, ${ }^{19}$ can be detected on the plasma membrane when overexpressed. ${ }^{20}$

Our double labelling experiments show that gliadin co-localises with HLA-DR antigens in late endosomes or lysosomes of small intestinal enterocytes from patients with untreated coeliac disease (Fig 2C). This labelling pattern could not be detected in control enterocytes. We assume that gliadin associates with HLADR antigens in late endosomes or lysosomes of enterocytes from patients with untreated coeliac disease, a prerequisite for the antigen presentation of gliadin to $\mathrm{T}$ lymphocytes and the immune processes in this condition. Because we can identify most of the HLA-DR antigens in late endosomes or lysosomes and the rare vacuoles containing gliadin without HLA-DR antigens, our results provide indirect evidence that gliadin and class II MHC proteins do not mainly associate at the apical surface or in early endosomes, but in a late endosomal or lysosomal compartment possibly after processing of gliadin. This view is supported by other studies. Antigen fragments can be detected in an immunologically relevant compartment after 30 minutes $^{21}$ and one hour passes between the initial exposure of antigen presenting cells to the antigen and the first stimulation of antigen sensitive $\mathrm{T}$ cells. ${ }^{22}$ In the human Epstein-Barr virus transformed B cell line, JY, a compartment enriched in class II molecules, showed lysosomal characteristics. ${ }^{8}$ Listeria monocytogenes are processed in endocytic vacuoles containing cathepsin D, Lamp 1, and class II MHC proteins but no $46 \mathrm{kD}$ mannose-6-phosphate receptors indicating their lysosomal nature. ${ }^{9}$ In contrast to this, immunocytochemical studies in the human B lymphoblastoid cell line, IM-9, involving investigation of cross linked surface immunoglobulins as intracellular markers comparable to multivalent antigens to $\mathrm{B}$ cells, ${ }^{23}$ suggest that early endosomes may be the site of antigen and class II MHC association. ${ }^{7}$

Which cellular biological mechanisms are involved in the translocation of gliadin to late endosomes or lysosomes of coeliac disease enterocytes is unknown. We suggest that the lack of association of ovalbumin and bovine serum albumin and the HLA-DR antigens of coeliac disease patients which we observed results from the fact that processed peptides from these proteins do not bind to HLA-DR antigens, unlike those of gliadin which do. This means that processed peptides of ovalbumin and bovine albumin cannot be routed to late endosomes and lysosomes. This process may be comparable with a ligand and its receptor whose intracellular pathway can change when the two bind. A receptor that binds gliadin on the apical membrane has not been isolated. IgA and IgG antibodies against gliadin and endomysium can be detected in the sera of almost all patients with untreated coeliac disease. The amounts of IgA and IgG are increased in the gut lumen of these patients. ${ }^{24}$ Internalised surface immunoglobulins were co-localised with class II molecules in lymphocytes. ${ }^{25} \mathrm{IgA}$ is known to be endocytosed and recycled at the apical surface of polarised epithelial cells after it has been transported by the polymeric immunoglobulin receptor from the basolateral membrane. Only about $5 \%$ can be transported from the apical membrane back to the basolateral membrane in Madin-Derby canine kidney cells expressing the polymeric immunoglobulin receptor. ${ }^{26}$ There is little evidence for receptor mediated transport of IgG across the intestinal epithelium: this has only been established in the neonatal rat. ${ }^{27} \mathrm{We}$ examined whether IgA might be involved in the translocation process of gliadin into late endosomes or lysosomes of enterocytes. Our detection of IgA in vacuoles enriched with class II MHC proteins or gliadin (Figs $3 \mathrm{~A}$ and $B$ ) indicates that IgA may reach a lysosomal compartment of epithelial cells under pathological conditions, as binding of a receptor to a polyvalent ligand could increase its down regulation. ${ }^{28}$ Alternatively, we cannot exclude the possibility that some gliadin molecules may already associate with HLA-DR antigens at the plasma membrane, after which they are transported to lysosomes. Whether binding of gliadin to IgA or the HLA-DR antigens on the apical surface of jejunal enterocytes contributes to the routing of gliadin to a lysosomal compartment remains to be explained.

Coeliac disease represents an interesting model for studying the endocytic transport of exogenous antigen to a compartment containing class II MHC proteins. This translocation process is necessary for the antigen presentation of gliadin and the immune reactions that 
result in this condition. The presentation of exogenous antigens by epithelial cells of the small intestine is suggested, but this remains to be proved. ${ }^{29} 30$ Exogenous antigen presentation has so far been shown to be restricted to lymphoid cells. ${ }^{5}$ Our results provide additional evidence in vivo, that exogenous antigen presentation may occur in non-lymphoid cells - that is, epithelial cells that have not been shown so far to present antigen to $T$ lymphocytes. Our experiments with control enterocytes show that gliadin is only present in vesicular and tubular structures of control enterocytes without any co-localisation with class II MHC proteins and cathepsin D. We speculate that gliadin is recycled back to the plasma membrane in control enterocytes in contrast to jejunal enterocytes of patients with untreated coeliac disease, where it is translocated to a late endosomal or lysosomal compartment enriched with class II MHC proteins. HLA-DR antigens are not colocalised with chicken egg and bovine albumin, two other nutrients. The localisation of gliadin to an HLA-DR antigen positive compartment of coeliac disease small intestinal enterocytes seems to be a specific translocation process. Whether this transport is mediated by antibodies originally directed against a peptide corresponding to amino acids $31-49$ of $\alpha$ gliadin, which has recently been shown to exacerbate coeliac disease using in vivo challenge feeding studies, is not known. ${ }^{31}$ The results described, however, suggest a role for epithelial cells of the small intestinal mucosa in the transport of gliadin to an HLA-DR positive compartment which precedes antigen presentation of gliadin to antigen sensitive T lymphocytes.

We thank Cordula Gebing for excellent technical assistance. This work was supported by the Deutsche Forschungsgemeinschaft (Zi 294/3-2, Zi 294/4-1, Zi 294/4-2).

1 Gjertsen HA, Lundin KEA, Sollid LM, Eriksen JA Thorsby E. T-cells recognise a peptide derived from $\alpha$-gliadin presented by the celiac disease-associated HLA-DO $\left(\alpha 1^{\star} 1501, \beta 1^{\star} 0201\right)$ heterodimer. Hum Immunol 1994; 39: 243-52.

2 Lundin KEA, Scott H, Hansen T, Paulsen G, Halstensen TS, Fausa O, et al. Gliadin specific HLA-DQ $\left(\mathrm{A} 1^{\star} 0501\right.$ $\mathrm{B} 1^{\star} 0201$ ) restricted T-cells isolated from the small intestinal mucosa of coeliac disease patients. $f$ Exp Med intestinal mucosa of

3 Kagnoff MF. Celiac disease. Gastroenterol Clin North Am 1992; 21: 3405-25.

4 Friis S, Dabelsteen E, Sjöström H, Norén O, Jarnum S Gliadin uptake in human enterocytes. Differences between coeliac patients in remission and control individuals. Gut 1992; 33: 1487-92.

5 Braciale TJ, Braciale VL. Antigen presentation: structural themes and functional variations. Immunol Today 1991; 12: 124-9.

6 Unanue ER. Cellular studies on antigen presentation by class II MHC molecules. Curr Opin Immunol 1992; 4: 63-9.

7 Guagliardi LE, Koppelman B, Blum JS, Marks MS, Cresswell P, Brodsky FM. Co-localization of molecules involved in antigen processing and presentation in an early endocytic compartment. Nature 1990; 343: 133-9.

8 Peters PJ, Nefjes JJ, Oorschot V, Ploegh HL, Geuze HJ. Segregation of MHC class II molecules form MHC class I molecules in the Golgi complex for transport to lysosomal compartments. Nature 1991; 349: to lyso $669-76$.

9 Harding CV, Geuze HJ. Class II MHC molecules are present in macrophage lysosomes and phagolysosomes that function in the phagocytic processing of Listeria monocytogenes for presentation of T-cells. $\mathcal{f}$ Cell Biol 1992; 119: 531-42.

10 Ciclitira PJ, Nelufer KM, Ellis HJ, Evans DJ. The effect of gluten on HLA-DR in the small intestinal epithelium of patients with coeliac disease. Clin Immunol 1986; 63: 101-4.

11 Scott H, Sollid LM, Brandtzaeg P, Thorsby E. Jejunal epithelium of patients with coeliac disease shows epithelium of patients with coeliac disease shows
enhanced expression of MHC II subregion products. $A d v$ Exp Med Biol 1988; 237: 689-93.

12 Fais S, Maiuri L, Pallone R, De Vincenzi M, De Ritis G, Troncone $R$, Auricchio $S$. Gliadin induced changes in the expression of MHC-class II antigens by human small intestinal epithelium. Organ culture studies with coeliac disease. Gut 1992; 33: 472-5.

13 Walker-Smith JA, Guandalini S, Schmitz J, Schmerling DM, Visakorpi JK. Revised criteria for diagnosis of coeliac disease. Arch Dis Child 1990; 65: 909-16.

14 Ciclitira PJ, Ellis HJ, Evans DJ, Lennox ES. Relation of antigenic structure of cereal proteins to their toxicity in coeliac patients. Br f Nutr 1985; 53: 39-45.

15 Tokuyasu KT. A study of positive staining of ultrathin frozen sections. Fournal of Ultrastructural Research 1978; 63: $287-307$.
frozen sections.

16 Griffiths G. Fine structure immunocytochemistry. Heidelberg: Springer-Verlag, 1993.

17 Zimmer KP, Hengst K, Carayon P, Bramswig J, Harms E. Different concentration of thyroid peroxidase and thyroglobulin in the nuclear envelope and the endoplasmic reticulum throughout the cytoplasm. Eur $f$ Cell Biol 1992; 57: 12-20.

18 Mayrhofer G, Spargo LDJ. Subcellular distribution of class II major histocompatibility antigens in enterocytes of the human and rat small intestine. Immunol Cell Biol 1989; 67: 251-60.

19 Green S, Zimmer KP, Griffiths G, Mellman I. Kinetics of intracellular transport and sorting of lysosomal membrane intracellular transport and sorting of lysosomal membrane and plasm

20 Harter C, Mellman I. Transport of the lysosomal membrane glycoprotein lgp120 (lgp-A) to lysosomes do not require appearance on the plasma membrane. $\mathcal{f}$ Cell Biol 1992; 117: 311-25.

21 Davidson HW, Watts C. Epitope-directed processing of specific antigen by B lymphocytes. $\mathcal{F}$ Cell Biol 1989; 109, 85-92.

22 Roosnek E, Demotz S, Corradin C, Lanzavecchia A Kinetics of MHC-antigen complex formation on antigenpresenting cells. $尹$ Immunol 1988; 140: 4079-82.

23 Myers CD, Kriz MK, Sunlivan TJ, Vitetta ES. Antigen-induced changes in phospholipid metabolism Antigen-induced changes in phospholipid metabolism in antigen

24 O'Mahony S, Arranz E, Barton JR, Ferguson A. Dissociation between systemic and mucosal humoral immune responses in coeliac disease. Gut 1991; 32: 29-35.

25 Pletscher M, Pernis B. Internalized membrane immunoglobulin meets intra-cytoplasmic DR antigen in human B lymphoblastoid cells. Eur $\mathcal{F}$ Immunol 1983; 13: 581-4.

26 Breitfeld PP, Harris JM, Mostov KE. Postendocytotic sorting of the ligand for the polymeric immunoglobulin receptor in Madin-Darby canine kidney cells. $f$ Cell Biol receptor in Madin-Dar

27 Rodewald R, Kraehenbuhl J-P. Receptor-mediated transport of IgG. $\mathcal{f}$ Cell Biol 1984; 99: 159S-64S.

28 Mellman I, Howe C, Helenius A. The control of membrane traffic on the endocytic pathway. Topics in Membranes Transport 1987; 29: 255-88.

29 Bland PW, Warren LG. Antigen presentation by epithelia cells of the rat small intestine. I. Kinetics, antigen specificity and blocking by anti-Ic antisera. Immunology 1986; 58: $1-7$.

30 Mayer L, Shlien R. Evidence for function of Ia molecules on gut epithelial cells in man. $f$ Exp Med 1987; 166: $1471-83$.

31 Sturgess RP, Day P, Ellis HJ, Lundin K, Gjertsen $H$, Kontakou M, Ciclitira PJ. Wheat peptide challenge in Kontakou M, Ciclitira PJ. Wheat peptide
coeliac disease. Lancet 1994; 343: 758-61. 